\title{
Downregulation of miR-485-3p promotes glioblastoma cell proliferation and migration via targeting RNF135
}

\author{
YE ZHANG, RUI SUI, YI CHEN, HAIYANG LIANG, JI SHI and HAOZHE PIAO \\ Department of Neurosurgery, Cancer Hospital of China Medical University, \\ Liaoning Cancer Hospital and Institute, Shenyang, Liaoning 110042, P.R. China
}

Received August 15, 2018; Accepted April 11, 2019

DOI: $10.3892 /$ etm.2019.7600

\begin{abstract}
MicroRNAs (miRNAs) are small non-coding RNAs that serve pivotal roles in human diseases. Several miRNAs, such as miR-485-3p, have been identified as potential biomarkers for predicting overall survival of patients with glioblastoma (GBM). However, the underlying mechanism of miRNAs in promoting GBM progression remains unknown. In the present study, decreased miR-485-3p expression was detected in tumor tissues from patients with GBM. Using western blot analysis, reverse transcription-quantitative PCR and dual luciferase reporter assay, ring finger protein 135 (RNF135) was confirmed as a target gene of miR-485-3p in GBM cells. Through silencing of RNF135, miR-485-3p inactivated the mitogen-activated protein kinase/ERK1/2 pathway in GBM cells. Moreover, functional assays demonstrated that miR-485-3p inhibited GBM cell proliferation and migration whilst overexpression of RNF135 reversed this effect. Additionally, a negative correlation between miR-485-3p and RNF135 mRNA expression was observed in tissues from patients with glioblastoma. In conclusion, the present results demonstrated that $\mathrm{miR}-485-3 \mathrm{p}$ functioned as a tumor suppressor which suggested that miR-485-3p might have a role in GBM progression.
\end{abstract}

\section{Introduction}

Glioblastoma (GBM) is the most commonly diagnosed type of malignant primary brain tumor (1). GBM is a high-grade tumor (World Health Organization grade IV), and prognosis of patients with GBM is relatively poor compared with other types of brain cancer (2). The median overall survival time is $<1$ year following diagnosis and the median progression-free survival

Correspondence to: Dr Haozhe Piao, Department of Neurosurgery, Cancer Hospital of China Medical University, Liaoning Cancer Hospital and Institute, 44 Xiaoheyan Road, Dadong, Shenyang, Liaoning 110042, P.R. China

E-mail: piaohaozhecmu@sina.com

Key words: microRNA-485-3p, glioblastoma, ring finger protein 135 is $\sim 6$ months $(3,4)$. Conventional therapeutic approaches for patients with GBM include chemotherapy, radiotherapy and surgical resection (5). Unfortunately, current treatments are unsatisfactory due to resistance development and cancer recurrence (6). Decades of study on the molecular mechanism of GBM have greatly enhanced our knowledge of GBM pathogenesis and provided new insights for development of targeted therapy (7). For example, it has been demonstrated that GBM cells with strong migration potential exhibit pro-apoptotic stimuli resistance (8) with several publications confirming that inhibitors that target migration-related molecules are effective methods to overcome resistance $(9,10)$. Therefore, further investigation into the molecular mechanism of GBM is still urgently required to meet clinical needs.

MicroRNAs (miRNAs) are a class of small non-coding RNAs first discovered in Caenorhabditis elegans (11). In many eukaryotic cells, miRNAs function as negative regulators of gene expression via directly binding to sites on the 3'untranslated region (UTR) of mRNAs (12). Dysregulation of miRNAs is implicated in the initiation and development of various types of cancer including GBM (13). Several miRNAs have been identified as oncogenes or tumor suppressors in GBM $(14,15)$. In addition, expression of certain miRNAs is a promising predictor of patient GBM outcome and therapy response $(16,17)$. miR-485-3p is mapped to the $14 \mathrm{q} 32.31$ chromosome region where mutations are frequently observed in cancers, which suggests that miR-485-3p might demonstrate tumor suppressor potential (18). A recent study determined that levels of miR-485-3p in serum could predict prognosis of patients with GBM (19). The focus of the present study was identifying the role and molecular mechanism of miR-485-3p in GBM cells.

Ring finger protein 135 (RNF135) is a RING finger domain-containing E3 ubiquitin ligase which has a critical role in many cellular biological processes via regulating protein degradation (20). For example, during viral infection, RNF135 ubiquitinates retinoic acid-inducible gene I to promote interferon- $\beta$ induction (21). Aberrant RNF135 expression results in altered expression of genes leading to several diseases $(22,23)$. In GBM, RNF135 functions as an oncogene through activating the mitogen-activated protein kinase (MAPK)/ERK pathway and controlling expression of key cell cycle regulators, such as cyclin dependent kinase 4, cyclin dependent kinase inhibitor $1 \mathrm{~A}$, cyclin dependent kinase 
inhibitor 1B (24). However, how RNF135 is regulated in GBM cells remains unknown; therefore, the present study aimed to determine the underlying mechanism.

\section{Materials and methods}

Collection of patient tissue. A total of 20 GBM and 20 normal tissues were collected at the Cancer Hospital of China Medical University between October 2014 to January 2017. The patients with GBM (15 males; 5 females; age, 25-48 years old) had undergone surgery, and patients with severe traumatic brain injury (13 males; 7 females; age, 23-56 years old) underwent internal decompression surgery. None of the patients sampled had undergone chemotherapy or radiotherapy prior to having surgery. Fresh tissue samples were histopathologically examined then immediately stored at $-80^{\circ} \mathrm{C}$ prior to RNA extraction. All patients provided written consent and the study was conducted under the supervision of the Ethics Committee of Cancer Hospital of China Medical University.

Bioinformatic analysis. Data for miR-485-3p expression levels in 5 normal tissues and 82 GBM tissues were downloaded from GSE25631 in the Gene Expression Omnibus database (https://www.ncbi.nlm.nih.gov/geo/). The comparison of miR-485-3p expression between normal and GBM tissues was conducted using GraphPad Prism v7.05 (GraphPad Software, Inc.).

U251-MG cell culture. The GBM cell line U251-MG was purchased from the American Type Culture Collection. The cells were cultured in Dulbecco's modified Eagle's medium (Gibco; Thermo Fisher Scientific, Inc.) containing 10\% fetal bovine serum (FBS; Hyclone; GE Healthcare Life Sciences) and $1 \%$ penicillin-streptomycin solution in a $37^{\circ} \mathrm{C}$ humidified incubator with $5 \% \mathrm{CO}_{2}$.

Reverse transcription-quantitative PCR (RT-qPCR). miRNeasy Mini kit (Qiagen, Inc.) was used to extract total RNA from patient tissues and U251-MG cells, following the manufacturer's instructions. The concentration and quality of extracted RNA was measured using NanoDrop 2000 (Thermo Fisher Scientific, Inc.). TransScript First-Strand cDNA Synthesis SuperMix (Transgen Biotech Co. Ltd.) was used to reverse transcribe RNA into cDNA, according to the manufacturer's protocol. qPCR was performed using SYBR Premix Ex Taq (Takara Bio, Inc.) on a CFX96 Touch $^{\mathrm{TM}}$ Real-Time PCR Detection System (Bio-Rad Laboratories, Inc.). The thermocycling conditions were: Predenaturing step at $95^{\circ} \mathrm{C}$ for $30 \mathrm{sec}$, followed by denaturing step at $95^{\circ} \mathrm{C}$ for $5 \mathrm{sec}$ then annealing and elongation at $60^{\circ} \mathrm{C}$ for $30 \mathrm{sec}$, repeated for 35 cycles. Gene expression was quantified using the $2^{-\Delta \Delta C q}$ method (25). The stem-loop method was used to detect miRNA expression. U6 and GAPDH were used as internal controls for miRNA and mRNA, respectively. Sequences of primers are listed in Table I.

Overexpression of $m i R-485-3 p$. miR-485-3p mimic and miR-negative control (NC) mimic were synthesized and purchased from Guangzhou RiboBio Co., Ltd. For the overexpression of miR-485-3p, $10 \mu \mathrm{lmiR}-485-3 \mathrm{p} \mathrm{mimic} \mathrm{(50} \mathrm{pmol} / \mu \mathrm{l})$
Table I. List of the primer sequences.

\begin{tabular}{ll}
\hline Primer & \multicolumn{1}{c}{ Sequence (5'-3') } \\
\hline Stem-loop primer & CTCAACTGGTGTCGTGGAGTCGG \\
miR-485-3p F & CAATTCAGTTGAGAGAGAGG \\
miR-485-3p R & CTCAACTGGTGTCGTGGA \\
RNF135 F & CTGCGGAAGAACACGCTACT \\
RNF135 R & GCTCAGTTCGTTGTCTGGTCC \\
U6 F & CTCGCTTCGGCAGCACA \\
U6 R & AACGCTTCACGAATTTGCGT \\
GAPDH F & CCACTCCTCCACCTTTGAC \\
GAPDH R & ACCCTGTTGCTGTAGCCA
\end{tabular}

miR, microRNA; RNF135, ring finger protein 135; F, forward; R, reverse.

was incubated with Lipofectamine 3000 (Invitrogen; Thermo Fisher Scientific, Inc.) in serum-free medium for $15 \mathrm{~min}$ and then added to each well in 96-well plates or 6-well plates. Experimentation commenced $48 \mathrm{~h}$ following transfection. The miR-485-3p mimic sequence was 5'-GUCAUACACGGCUCU CCUCUCU-3' and the miR-NC mimic sequence was 5'-UUC UCCGAACGUGUCACGUTT-3'.

Western blot analysis. Cell lysates were prepared using radioimmunoprecipitation lysis buffer (Beyotime Institute of Biotechnology). The concentration of protein lysate was determined with Pierce Bicinchoninic Acid Protein Assay kit (Thermo Fisher Scientific, Inc.). A total of $20 \mu \mathrm{g}$ protein were loaded per lane and separated via SDS-PAGE on an $8 \%$ gel. The separated proteins were then transferred to polyvinylidene difluoride membranes and subsequently blocked with $5 \%$ non-fat milk at room temperature for $1 \mathrm{~h}$. Then membranes were incubated with primary antibodies anti-RNF135 (cat. no. ab28636; 1:1,000; Abcam), anti-GAPDH (cat. no. G8795; 1:8,000; Sigma-Aldrich; Merck KGaA), ERK1/2 (cat. no. 4695; 1:1,000; Cell Signaling Technology, Inc.) and phosphorylated (p-) ERK1/2 (cat. no. 9101; 1:1,000; Cell Signaling Technology, Inc.) at $4^{\circ} \mathrm{C}$ overnight. Following primary incubation, membranes were incubated with horseradish peroxidase-labeled secondary mouse antibody (cat. no. AP308P; 1:10,000; Sigma-Aldrich; Merck KGaA) and rabbit antibody (cat. no. SAB3700852; 1:10,000; Sigma-Aldrich; Merck $\mathrm{KGaA}$ ) at room temperature for $1 \mathrm{~h}$. Subsequently, the bands were visualized using enhanced chemiluminescence western blotting detection reagents (GE Healthcare Life Sciences). Images were captured and analyzed using ImageQuant TL 7.0 (GE Healthcare Life Sciences). GAPDH served as a loading control for protein quantification.

Dual luciferase reporter assay. To investigate whether miR-485-3p directly regulated RNF135, bioinformatic analysis (TargetScan V7.2; www.targetscan.org) was performed to compare the miR-485-3p sequence with the 3'UTR of RNF135 mRNA. Dual luciferase assay was performed using the Dual-Luciferase ${ }^{\circledR}$ Reporter Assay System (Promega 
A

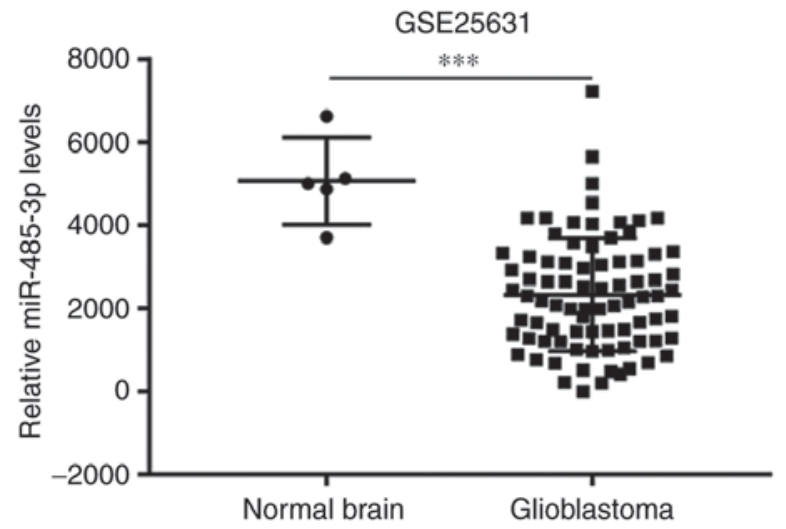

B

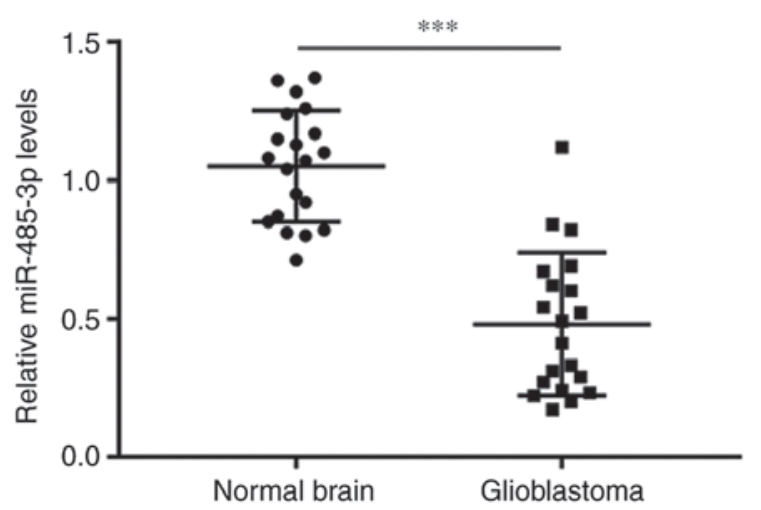

Figure 1. Expression of miR-485-3p in glioblastoma tissues. (A) Expression data for miR-485-3p were downloaded from the GSE25631 dataset which contained 5 normal brain tumor tissues and 82 glioblastoma tissues. Compared with normal tissues, miR-485-3p was downregulated in glioblastoma tissues. (B) Expression of miR-485-3p was decreased in 20 glioblastoma tissues compared with 20 normal brain tissues. ${ }^{* * *} \mathrm{P}<0.001$, with comparisons indicated by lines. miR, microRNA.

A

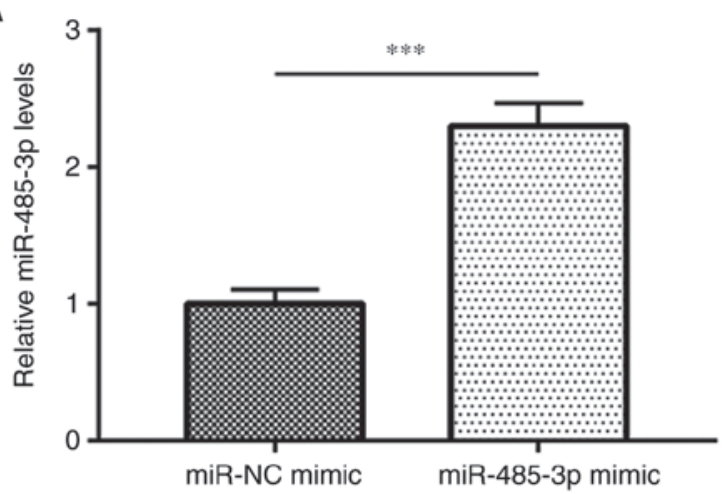

B

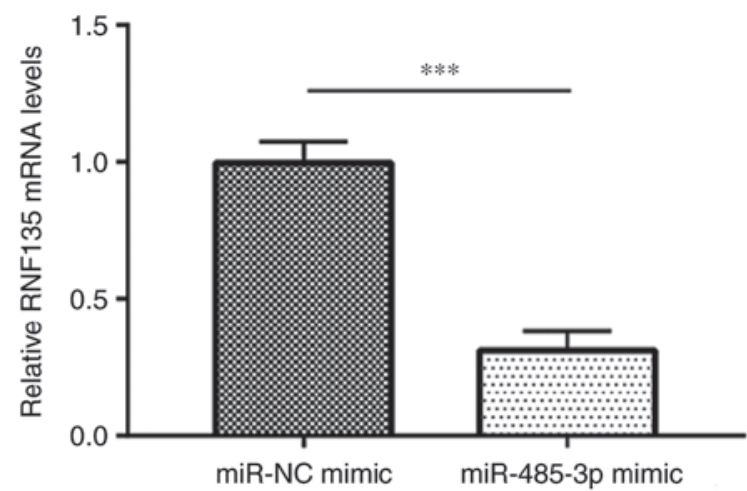

C
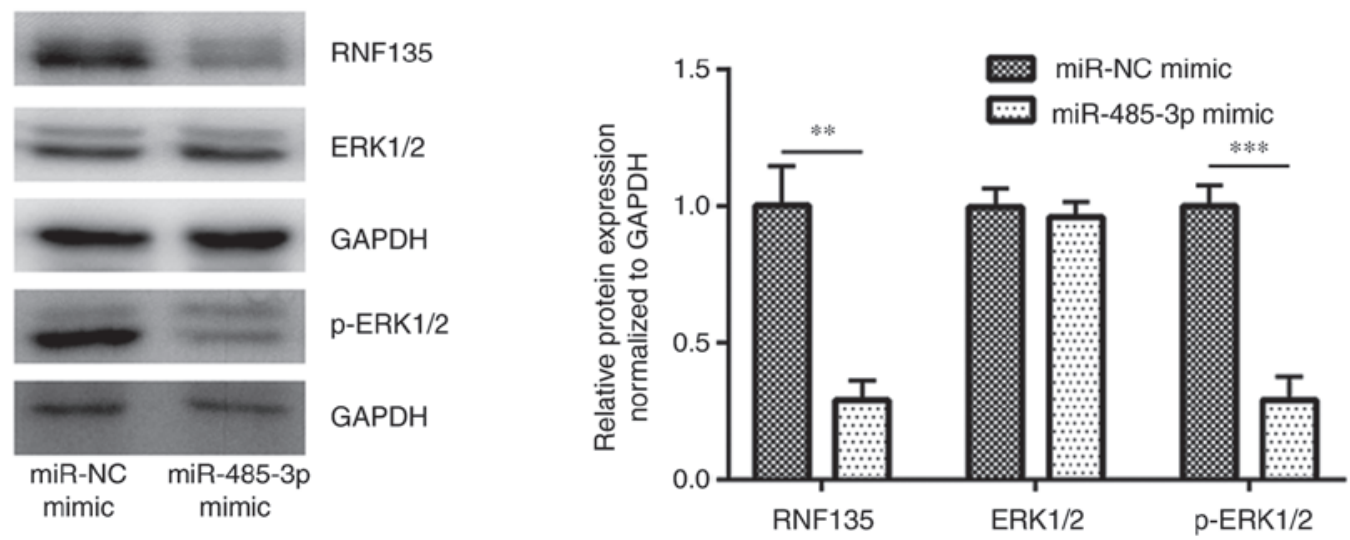

Figure 2. Overexpression of miR-485-3p decreases RNF135 expression in glioblastoma cells. (A) Transfection of miR-485-3p mimic increased miR-485-3p levels in U251-MG cells. (B) Overexpression of miR-485-3p decreased RNF135 mRNA levels in U251-MG cells. (C) Representative western blots and quantification demonstrated that overexpression of miR-485-3p decreased RNF135 and p-ERK1/2 protein levels in U251-MG cells. ${ }^{* *} \mathrm{P}<0.01 ;{ }^{* * * *} \mathrm{P}<0.001$, with comparisons indicated by lines. miR, microRNA; RNF135, ring finger protein 135; p-, phosphorylated; NC, negative control.

Corporation). U251-MG cDNA was prepared by isolating RNA from U251-MG cells using TRIzol reagent (Invitrogen; Thermo Fisher Scientific, Inc.) followed by reverse-transcription into first-strand cDNA using RevertAid RT Reverse Transcription kit (Thermo Fisher Scientific, Inc.) according to manufacturer's protocol. The 3'UTR containing putative binding sites of miR-485-3p on RNF135 mRNA was amplified from the U251-MG cDNA and ligated into pGL3-basic vector (Addgene, Inc.) using XbaI enzyme (New England Biolabs, Inc.). The primer sequences of the RNF135 mRNA were: forward, 5'-CTCTAGACCTATCGCTGGAGCTGT GAG-3' and reverse, 5'-CTCTAGAAGGAATTCGAC ACCAGCCTG-3'. 3'UTR mutant (Mut) 1 and Mut 2 were constructed by introducing 2 site mutations into putative binding site 1 and putative binding site 2 respectively with Q5 ${ }^{\circledast}$ Site-Directed Mutagenesis kit (New England BioLabs, 
Inc.). In brief, U251-MG cells were cultured in 24-well plates and co-transfected with miR-485-3p mimic or miR-NC mimic with pGL3-RNF135 3'UTR-wild type (WT) or pGL3-RNF135 3'UTR-Mut 1 or pGL3-RNF135 3'UTR-Mut 2 and pRL-TK vector (Promega Cooperation) using Lipofectamine 3000 (Invitrogen; Thermo Fisher Scientific, Inc.). Following 48 h, cells were collected and the dual-luciferase activity was examined using Dual Luciferase Reporter Assay system (Promega Cooperation) following the manufacturer's protocol with Renilla luciferase as the internal control.

Construction of plasmid and overexpression of RNF135. The full length RNF135 cDNA from U251-MG cells was cloned into pcDNA3.1 plasmid (Addgene, Inc.) to construct the pcDNA3.1-RNF135 plasmid. For overexpression of RNF135 with or without overexpression of miR-485-3p, $2 \mu \mathrm{g}$ pcDNA3.1-RNF135 and/or $10 \mu \mathrm{lmiR}-485-3 \mathrm{p}$ mimics $(50 \mathrm{pmol} / \mu \mathrm{l})$ were transfected into U251-MG cells using Lipofectamine 3000 (Invitrogen; Thermo Fisher Scientific, Inc.) according to manufacturer's protocol.

Cell proliferation assay. Cell proliferation was measured using Cell Counting kit-8 (CCK-8; Dojindo Molecular Technologies, Inc.) according to manufacturer's protocol. In brief, 2,000 U251-MG cells were seeded into 96-well plates then incubated under standard conditions. The next day, cells were transfected with pcDNA3.1-RNF135 with or without miR-485-3p mimic. At $0,24,48$ and $72 \mathrm{~h}$ post transfection, $10 \mu \mathrm{l}$ CCK- 8 solution was added to each well and the cells were incubated for another $2 \mathrm{~h}$ at $37^{\circ} \mathrm{C}$. The absorbance at $450 \mathrm{~nm}$ was detected using a microplate reader (Bio-Rad Laboratories, Inc.) to determine the number of cells.

Cell migration assay. Cell migration ability was detected using a wound-healing assay. U251-MG cells $\left(1 \times 10^{6}\right)$ were seeded into 6-well plates and cultured under standard conditions until $90 \%$ confluence. The cells were then transfected with miR-485-3p mimic with or without pcDNA3.1-RNF135. The following day, a wound area was made by scratching the center of each well with a $10 \mu 1$ pipette tip. The wells were washed with PBS then culture medium containing 1\% FBS was added. At 0 and $20 \mathrm{~h}$, images of the scratch area were captured. Subsequently, the percentage of the relative wound closure area was analyzed using Image Pro Plus 6 (Media Cybernetics, Inc.).

Statistical analysis. All statistical analyses were carried out using GraphPad Prism 7 software (GraphPad Software, Inc.). Data were presented as the mean \pm standard deviation. Student's t-test was used to compare 2 groups and multiple groups were compared using one-way analysis of variance followed by the Newman-Keuls post hoc test. $\mathrm{P}<0.05$ was considered to indicate statistical significance.

\section{Results}

miR-485-3p is downregulated in GBM compared with normal brain tissues. Decreased expression of miR-485-3p has been identified in several cancer types $(26,27)$ including GBM $(19)$. To evaluate the expression of miR-485-3p in GBM tissues,

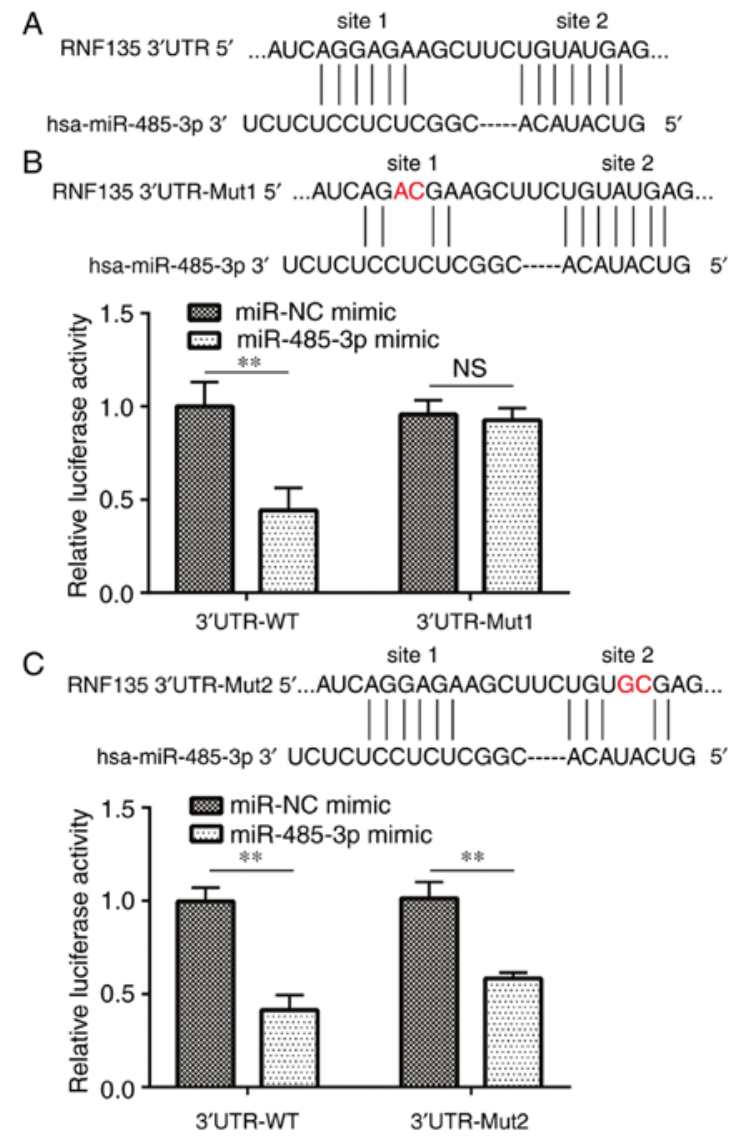

Figure 3. RNF135 is a target gene of miR-485-3p in glioblastoma cells. (A) Comparison of the sequences of miR-485-3p and 3'UTR of RNF135 mRNA demonstrated that there were two potential binding sites for miR-485-3p on RNF135 3'UTR. (B) Dual luciferase reporter assay demonstrated that miR-485-3p mimic decreased luciferase activity of RNF135 3'UTR-WT but not RNF135 3'UTR-Mut1 in U251-MG cells. (C) Dual luciferase reporter assay demonstrated that miR-485-3p mimic decreased luciferase activity of both RNF135 3'UTR-WT and RNF135 3'UTR-Mut2 in U251-MG cells. Mutated nucleotides are highlighted in red font. ${ }^{* *} \mathrm{P}<0.01$, with comparisons indicated by lines. RNF135, ring finger protein 135; miR, microRNA; UTR, untranslated region; WT, wild-type; MUT, mutant; NS, not significant.

miR-485-3p expression data was downloaded from GSE25631 which contained miRNA microarray data for 5 normal brain tissues and 82 GBM tissues. Compared with the normal brain group, miR-485-3p levels were significantly decreased in GBM tissues (Fig. 1A). To further validate this finding, RT-qPCR was performed to compare miR-485-3p expression in $20 \mathrm{GBM}$ tissues and 20 normal brain tissues collected at our institution. Significant downregulation of miR-485-3p was detected in GBM tissues compared with normal brain tissues (Fig. 1B), which suggested that miR-485-3p may have a tumor suppressor role in GBM.

Overexpression of miR-485-3p decreases RNF135 expression and inactivates the MAPK/ERK pathway in GBM cells. RNF135 is a recently identified GBM oncogene (24). Transfection of miR-485-3p mimic increased miR-485-3p expression in U251-MG cells (Fig. 2A), accompanied with a decreased expression of RNF135 mRNA (Fig. 2B). Western blot analysis demonstrated that overexpression of miR-485-3p also decreased RNF135 protein expression (Fig. 2C). Overactivation 


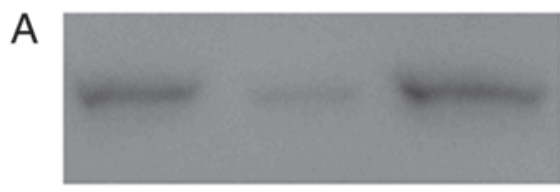

RNF135

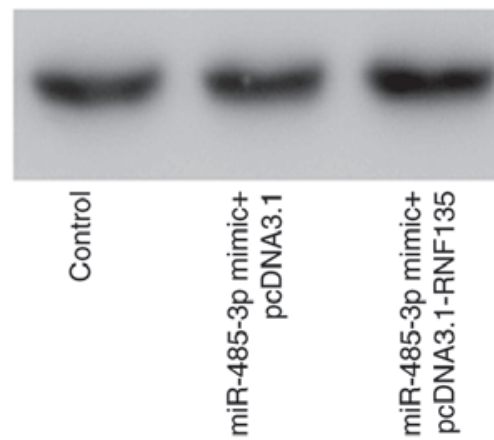

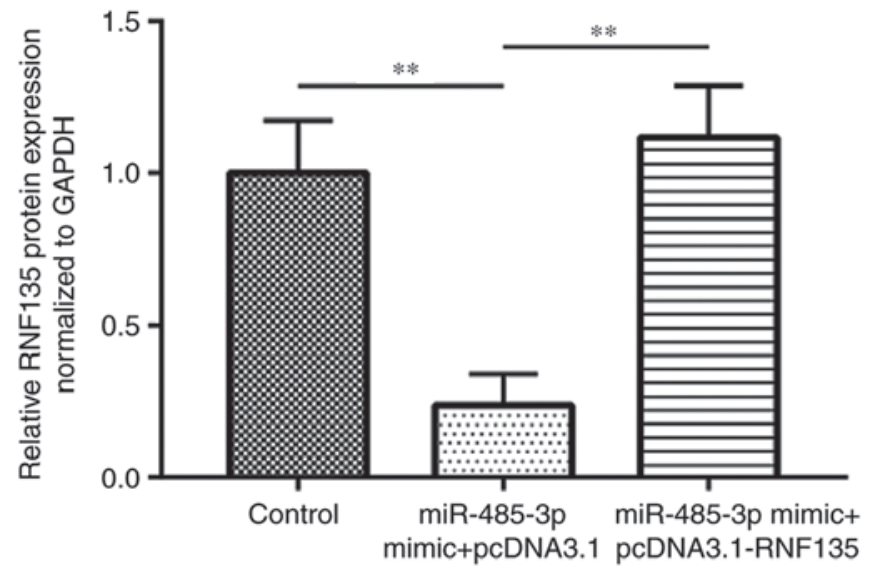

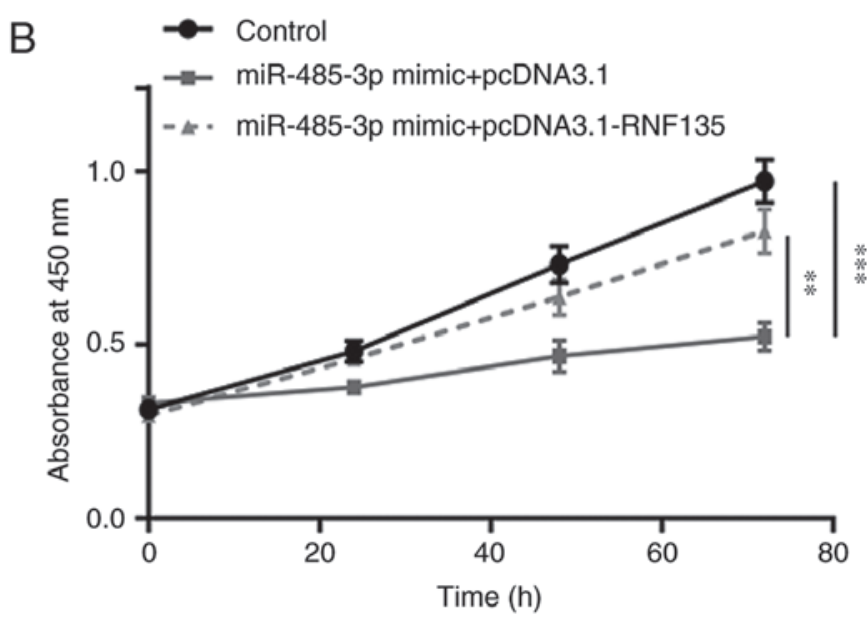

Figure 4. Overexpression of miR-485-3p inhibits glioblastoma cell growth through repression of RNF135. (A) Representative images and quantification from western blot analysis. Transfection with miR-485-3p mimic reduced RNF135 protein expression, while transfection with a RNF135-expressing plasmid reversed this effect in U251-MG cells. (B) Overexpression of miR-485-3p inhibited cell proliferation while transfection of recombinant RNF135 partially recovered cell growth in U251-MG cells. ${ }^{* *} \mathrm{P}<0.01 ;{ }^{* * * *} \mathrm{P}<0.001$, with comparisons indicated by lines. miR, microRNA; RNF135, ring finger protein 135 .

of MAPK/ERK signaling promotes GBM progression, which is regulated by RNF135 (21). In addition to downregulation of RNF135, overexpression of miR-485-3p also decreased p-ERK1/2 levels, indicating an inactivation of MAPK/ERK signaling (Fig. 2C). These results suggested that miR-485-3p negatively regulated RNF135 to inhibit GBM cells.

RNF135 is a target gene of miR-485-3p. There were two putative binding sites between miR-485-3p and 3'UTR of RNF135 (Fig. 3A). Dual luciferase reporter assays demonstrated that overexpression of miR-485-3p significantly decreased luciferase activity of RNF135 3'UTR-WT in U251-MG cells (Fig. 3B and C). Additionally, miR-485-3p mimic induced decreased luciferase activity of RNF135 3'UTR-Mut2 but not RNF135 3'UTR-Mut1, which suggested that site 1 was a direct binding site for miR-485-3p on the 3'UTR of RNF135 in GBM cells (Fig. 3B and C).

Overexpression of miR-485-3p inhibits GBM cell proliferation and migration via targeting RNF135. Recombinant RNF135 was constructed and co-transfected with miR-485-3p mimic to study the functions of miR-485-3p/RNF135 in GBM cells.
Transfection with miR-485-3p mimic decreased RNF135 protein expression whereas co-transfection of recombinant RNF135 and miR-485-3p mimic rescued RNF135 expression in U251-MG cells (Fig. 4A). In the cell proliferation assay, overexpression of miR-485-3p decreased the cell viability compared with control, whilst overexpression of RNF135 partially recovered the cell viability (Fig. 4B). These results suggested that miR-485-3p inhibited GBM cell proliferation via repression of RNF135. In the cell migration assay, overexpression of miR-485-3p inhibited cell migration. Transfection with recombinant RNF135 attenuated this inhibitory effect (Fig. 5A and B). These results demonstrated that miR-485-3p inhibited GBM cell proliferation and migration via repression of RNF135.

Expression of miR-485-3p is negatively correlated with RNF135 in GBM tissues. Next, the potential association between miR-485-3p and RNF135 in GBM tissues was investigated. RT-qPCR was performed on the $20 \mathrm{GBM}$ and 20 normal tissues collected in the present study, to detect RNF135 mRNA levels. Significant upregulation of RNF135 mRNA levels were observed in GBM tissues compared with normal tissues (Fig. 6A). Crucially, Pearson correlation 
A
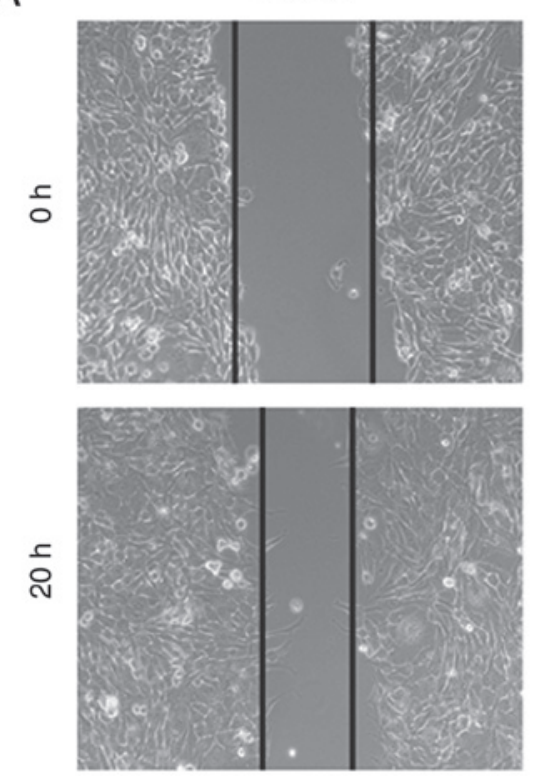

miR-485-3p mimic+pcDNA3.1
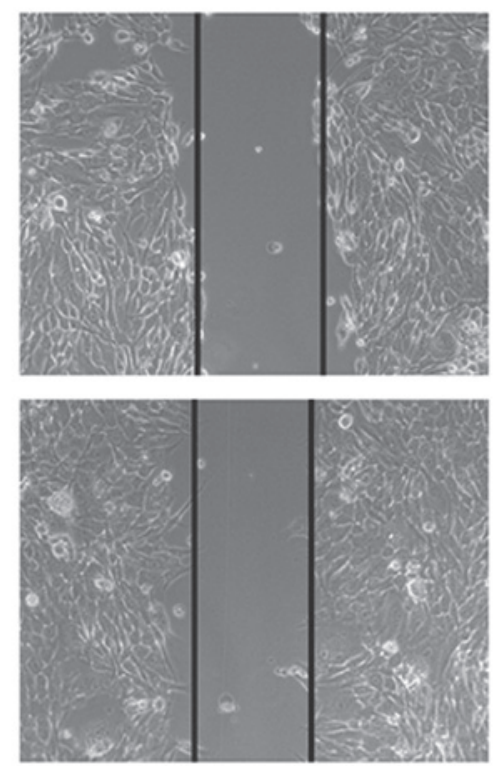

miR-485-3p mimic+pcDNA3.1-RNF135
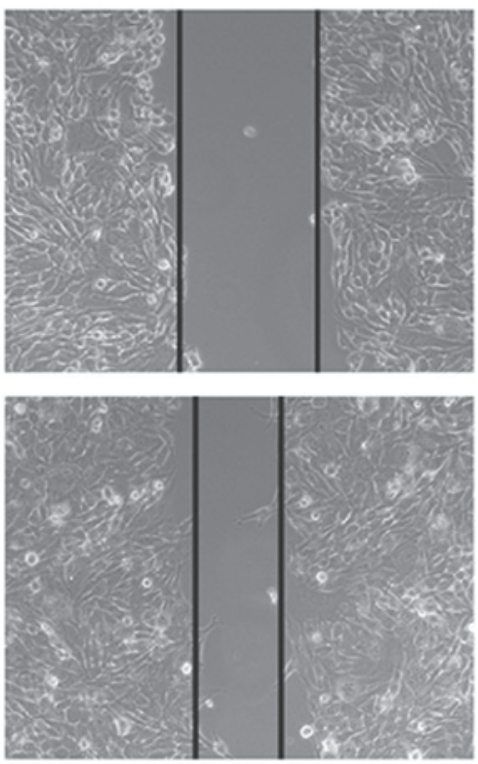

B

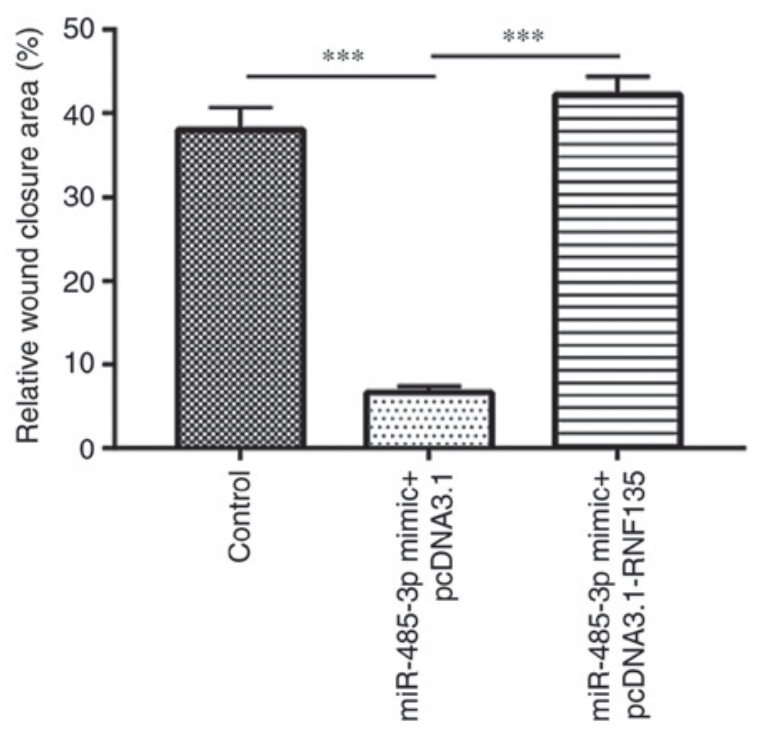

Figure 5. Overexpression of miR-485-3p inhibits glioblastoma cell migration through repression of RNF135. (A) Micrographs captured at 0 and $20 \mathrm{~h}$ of the wound-healing assay (magnification, x100). Overexpression of miR-485-3p inhibited cell migration whilst transfection of recombinant RNF135 reversed cell migration inhibition in U251-MG cells. (B) Quantification of relative wound closure area. ${ }^{* * *} \mathrm{P}<0.001$, with comparisons indicated by lines. miR, microRNA; RNF135, ring finger protein 135 .

A

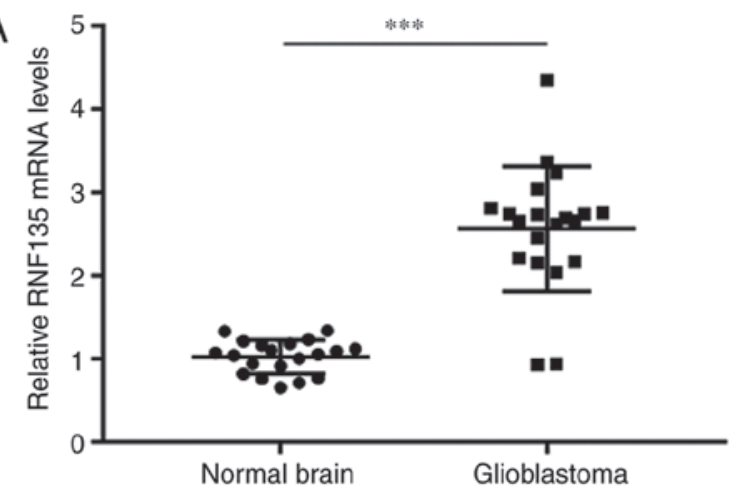

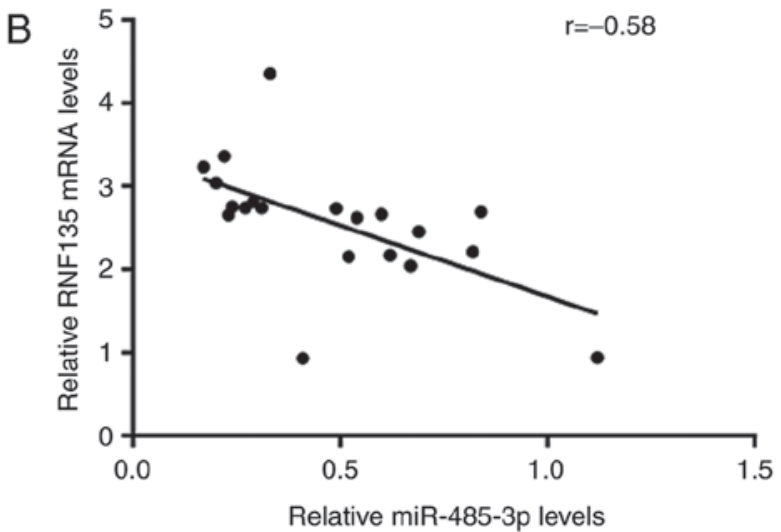

Figure 6. RNF135 is overexpressed in glioblastoma tissues and its expression is negative correlated with miR-485-3p levels. (A) Expression of RNF135 mRNA was increased in glioblastoma tissues compared with tissue from normal brains. (B) RNF135 mRNA levels were negatively correlated with miR-485-3p levels. ${ }^{* * *} \mathrm{P}<0.001$, with comparisons indicated by lines. RNF135, ring finger protein $135 ;$ miR, microRNA. 
analysis demonstrated that miR-485-3p expression was negatively correlated with RNF135 mRNA levels in GBM tissues $(\mathrm{r}=-0.58 ; \mathrm{P}<0.01$; Fig. 6B).

\section{Discussion}

GBM is a lethal cancer type (28) and aberrant expression of miRNAs is experimentally identified as a major step towards development of the disease (29). miR-485-3p, mapped to chromosome 14q32.31 region, is a well-characterized tumor suppressor in several cancer types (30). Recently, miRNA microarray data of 14 GBM tissues identified miR-485-3p as one of the significantly downregulated miRNAs in tissues from short-survival patients compared with long-survival patients (31). Low expression of serum miR-485-3p predicts poor overall survival in patients with GBM (19). The present study determined that miR-485-3p was downregulated in GBM. RT-qPCR and western blot analysis demonstrated that RNF-135 was negatively regulated by miR-485-3p. Dual luciferase assay confirmed RNF135 as a target gene of miR-485-3p. Functional assays indicated that miR-485-3p inhibited cell proliferation and migration of GBM cells via repression of RNF135. The present findings suggested that miR-485-3p was a tumor suppressor in GBM.

In order to investigate the role of miR-485-3p in GBM, analysis of miRNA expression in normal brain tissues and GBM tissues from a previously published microarray dataset was performed. Results determined that miR-485-3p expression was significantly lower in GBM tissues compared with normal tissues. To validate this result, the present study collected and analyzed 20 pairs of normal brain and GBM tissues. Therefore, in addition to the reported decreased miR-485-3p expression in serum and tissues of short-survival patients $(19,31)$, the present study identified miR-485-3p as a downregulated miRNA in GBM tissues compared with normal brain tissues. In addition, functional analysis of miR-485-3p demonstrated that miR-485-3p overexpression inhibited cell proliferation and migration of GBM cells. GBM is highly aggressive with cells displaying strong proliferative capacity (28) therefore the current findings suggested that miR-485-3p may be involved in the development of GBM.

E3 ubiquitin ligases regulate turnover of many target genes via protease degradation (32). In GBM, dysregulation of E3 ligases results in accumulation of oncogenes or downregulation of tumor suppressors, leading to GBM progression $(33,34)$. RNF135 is a member of E3 ubiquitin ligases and regulates protein stability (24). High expression of RNF135 is associated with poor prognosis for GBM patients (24). RNF135 activates the MAPK/ERK signaling pathway and facilitates progression of the GBM cell cycle to promote proliferation (24). The present study identified that overexpression of miR-485-3p decreased RNF135 expression in GBM cells. Bioinformatic analysis and the dual luciferase reporter assay predicted and confirmed RNF135 as a target gene of miR-485-3p. In GBM tissues, a significant negative correlation between RNF135 mRNA and miR-485-3p expression was identified. RNF135 function has been previously reported to be regulated by mutation $(20,35)$. The present findings demonstrated that RNF135 was also regulated by miRNA. Crucially, cell proliferation and migration inhibition induced by $\mathrm{miR}-485-3 \mathrm{p}$ mimic was reversed by overexpression of RNF135, which indicated that miR-485-3p exerted its tumor suppressive function through RNF135.

In conclusion, the present study provided evidence to suggest that miR-485-3p may have a role as a tumor suppressor in GBM via regulation of RNF135.

\section{Acknowledgements}

Not applicable.

\section{Funding}

The study was funded by the Clinical Capability Construction Project for Liaoning Provincial Hospitals (grant no. LNCCC-B04-2015).

\section{Availability of data and materials}

The datasets generated and/or analyzed during the current study are available from the corresponding author on reasonable request.

\section{Authors' contributions}

Clinical sample collection was performed by JS and HL. The study was designed by HP and RS. Data acquisition and analysis were performed by HP, YZ and YC. The manuscript was prepared, edited and reviewed by HP and JS.

\section{Ethics approval and consent to participate}

All patients provided written informed consent and the Ethic Committee of Cancer Hospital of China Medical University approved the present study.

\section{Patient consent for publication}

Not applicable.

\section{Competing interests}

The authors declare that they have no competing interests.

\section{References}

1. Siegel RL, Miller KD and Jemal A: Cancer statistics, 2018. CA Cancer J Clin 68: 7-30, 2018.

2. Wick W and Platten M: Understanding and treating glioblastoma. Neurol Clin 36: 485-499, 2018.

3. Buckner JC: Factors influencing survival in high-grade gliomas. Semin Oncol 30: 10-14, 2003.

4. Das S and Marsden PA: Angiogenesis in glioblastoma. N Engl J Med 369: 1561-1563, 2013.

5. Fine HA: The basis for current treatment recommendations for malignant gliomas. J Neurooncol 20: 111-120, 1994.

6. Wick W, Osswald M, Wick A and Winkler F: Treatment of glioblastoma in adults. Ther Adv Neurol Disord 11: $1756286418790452,2018$.

7. Nguyen HS, Shabani S, Awad AJ, Kaushal M and Doan N: Molecular markers of therapy-resistant glioblastoma and potential strategy to combat resistance. Int J Mol Sci 19: pii: E1765, 2018. 
8. Osswald M, Jung E, Sahm F, Solecki G, Venkataramani V, Blaes J, Weil S, Horstmann H, Wiestler B, Syed M, et al: Brain tumour cells interconnect to a functional and resistant network. Nature 528: 93-98, 2015.

9. Lefranc F, Le Rhun E, Kiss R and Weller M: Glioblastoma quo vadis: Will migration and invasiveness reemerge as therapeutic targets? Cancer Treat Rev 68: 145-154, 2018.

10. Onken J, Torka R, Korsing S, Radke J, Krementeskaia I, Nieminen M, Bai X, Ullrich A, Heppner F and Vajkoczy P: Inhibiting receptor tyrosine kinase AXL with small molecule inhibitor BMS-777607 reduces glioblastoma growth, migration and invasion in vitro and in vivo. Oncotarget 9: 9876-9889, 2016

11. Lee RC, Feinbaum RL and Ambros V: The C. elegans heterochronic gene lin-4 encodes small RNAs with antisense complementarity to lin-14. Cell 75: 843-854, 1993.

12. Bartel DP: MicroRNAs: Genomics, biogenesis, mechanism and function. Cell 116: 281-297, 2004.

13. Sumazin $P$, Yang X, Chiu HS, Chung WJ, Iyer A, Llobet-Navas D, Rajbhandari P, Bansal M, Guarnieri P, Silva J and Califano A: An extensive microRNA-mediated network of RNA-RNA interactions regulates established oncogenic pathways in glioblastoma. Cell 147: 370-381, 2011

14. Yue S, Wang L, Zhang H, Min Y, Lou Y, Sun H, Jiang Y, Zhang W, Liang A, Guo Y, et al: miR-139-5p suppresses cancer cell migration and invasion through targeting ZEB1 and ZEB2 in GBM. Tumour Biol 36: 6741-6749, 2015.

15. Zhou X, Ren Y, Moore L, Mei M, You Y, Xu P, Wang B, Wang G, Jia Z, Pu P, et al: Downregulation of miR-21 inhibits EGFR pathway and suppresses the growth of human glioblastoma cells independent of PTEN status. Lab Invest 90: 144-155, 2010.

16. Li W, Guo F, Wang P, Hong S and Zhang C: miR-221/222 confers radioresistance in glioblastoma cells through activating Akt independent of PTEN status. Curr Mol Med 14: 185-195, 2014.

17. Xiong X, Deng J, Zeng C, Jiang Y, Tang S and Sun X: MicroRNA-141 is a tumor regulator and prognostic biomarker in human glioblastoma. Oncol Lett 14: 4455-4460, 2017.

18. Avet-Loiseau H, Facon T, Grosbois B, Magrangeas F, Rapp MJ, Harousseau JL, Minvielle S and Bataille R; Intergroupe Francophone du Myélome: Oncogenesis of multiple myeloma: $14 q 32$ and $13 q$ chromosomal abnormalities are not randomly distributed, but correlate with natural history, immunological features, and clinical presentation. Blood 99: 2185-2191, 2002.

19. Wang ZQ, Zhang MY, Deng ML, Weng NQ, Wang HY and Wu SX: Low serum level of miR-485-3p predicts poor survival in patients with glioblastoma. PLoS One 12: e0184969, 2017.

20. Tastet J, Decalonne L, Marouillat S, Malvy J, Thépault RA, Toutain A, Paubel A, Tabagh R, Bénédetti H,Laumonnier F, et al: Mutation screening of the ubiquitin ligase gene RNF135 in French patients with autism. Psychiatr Genet 25: 263-267, 2015.

21. Oshiumi H, Matsumoto M, Hatakeyama $S$ and Seya $T$ : Riplet/RNF135, a RING finger protein, ubiquitinates RIG-I to promote interferon-beta induction during the early phase of viral infection. J Biol Chem 284: 807-817, 2009.

22. Jin J, Zhao L and Li Z: The E3 ubiquitin ligase RNF135 regulates the tumorigenesis activity of tongue cancer SCC25 cells. Cancer Med 5: 3140-3146, 2016.
23. Tastet J, Decalonne L, Marouillat S, Malvy J, Thépault RA, Toutain A, Paubel A, Tabagh R, Bénédetti H, Laumonnier F, et al: Mutation screening of the ubiquitin ligase gene RNF135 in French patients with autism. Psychiat Genet 6: 263-267, 2015.

24. Liu Y, Wang F, Liu Y, Yao Y, Lv X, Dong B, Li J, Ren S, Yao Y and $\mathrm{Xu}$ Y: RNF135, RING finger protein, promotes the proliferation of human glioblastoma cells in vivo and in vitro via the ERK pathway. Sci Rep 6: 20642, 2016.

25. Livak KJ and Schmittgen TD: Analysis of relative gene expression data using real-time quantitative PCR and the 2(-Delta Delta C(T)) method. Methods 25: 402-408, 2001

26. Lou C, Xiao M, Cheng S, Lu X, Jia S, Ren Y and Li Z: MiR-485-3p and miR-485-5p suppress breast cancer cell metastasis by inhibiting PGC-1 $\alpha$ expression. Cell Death Dis 7: e2159, 2016.

27. Xiong D, Sheng Y, Ding S, Chen J, Tan X, Zeng T, Qin D, Zhu L, Huang A and Tang H: LINC00052 regulates the expression of NTRK 3 by miR-128 and miR-485-3p to strengthen HCC cells invasion and migration. Oncotarget 7: 47593-47608, 2016.

28. Batash R, Asna N, Schaffer P, Francis N and Schaffer M: Glioblastoma multiforme, diagnosis and treatment; recent literature review. Curr Med Chem 24: 3002-3009, 2017.

29. Ahir BK, Ozer H, Engelhard HH and Lakka SS: MicroRNAs in glioblastoma pathogenesis and therapy: A comprehensive review. Crit Rev Oncol Hematol 120: 22-33, 2017.

30. Formosa A, Markert EK, Lena AM, Italiano D, Finazzi-Agro' E, Levine AJ, Bernardini S, Garabadgiu AV, Melino G and Candi E: MicroRNAs, miR-154, miR-299-5p, miR-376a, miR-376c miR-377, miR-381, miR-487b, miR-485-3p, miR-495 and miR-654-3p, mapped to the 14q32.31 locus, regulate proliferation, apoptosis, migration and invasion in metastatic prostate cancer cells. Oncogene 33: 5173-5182, 2014.

31. Henriksen M, Johnsen KB, Olesen P, Pilgaard L and Duroux M: MicroRNA expression signatures and their correlation with clinicopathological features in glioblastoma multiforme. Neuromolecular Med 16: 565-577, 2014.

32. Sun Y: Targeting E3 ubiquitin ligases for cancer therapy. Cancer Biol Ther 2: 623-629, 2003.

33. Xu T, Wang H, Jiang M, Yan Y, Li W, Xu H, Huang Q, Lu Y and Chen J: The E3 ubiquitin ligase CHIP/miR-92b/PTEN regulatory network contributes to tumorigenesis of glioblastoma. Am J Cancer Res 7: 289-300, 2017.

34. Qi Z, Cai S, Cai J, Chen L, Yao Y, Chen L and Mao Y: miR-491 regulates glioma cells proliferation by targeting TRIM28 in vitro. BMC Neurol 16: 248, 2016.

35. Douglas J, Cilliers D, Coleman K, Tatton-Brown K, Barker K, Bernhard B, Burn J, Huson S, Josifova D, Lacombe D, et al: Mutations in RNF135, a gene within the NF1 microdeletion region, cause phenotypic abnormalities including overgrowth. Nat Genet 39: 963-965, 2007.

This work is licensed under a Creative Commons Attribution-NonCommercial-NoDerivatives 4.0 International (CC BY-NC-ND 4.0) License. 